\title{
Revisiting an Old Concept: The Gradient Wind*
}

\author{
KEITH F. BRILL \\ Weather Prediction Center, National Centers for Environmental Prediction, College Park, Maryland
}

(Manuscript received 15 March 2013, in final form 20 June 2013)

\begin{abstract}
The gradient wind is defined as a horizontal wind having the same direction as the geostrophic wind but with a magnitude consistent with a balance of three forces: the pressure gradient force, the Coriolis force, and the centrifugal force arising from the curvature of a parcel trajectory. This definition is not sufficient to establish a single way of computing the gradient wind. Different results arise depending upon what is taken to be the parcel trajectory and its curvature. To clarify these distinctions, contour and natural gradient winds are defined and subdivided into steady and nonsteady cases. Contour gradient winds are based only on the geostrophic streamfunction. Natural gradient winds are obtained using the actual wind. Even in cases for which the wind field is available along with the geostrophic streamfunction, it may be useful to obtain the gradient wind for comparison to the existing analyzed or forecast wind or as a force-balanced reference state. It is shown that the nonanomalous (normal) solution in the case of nonsteady natural gradient wind serves as an upper bound for the actual wind speed. Otherwise, supergradient wind speeds are possible, meaning that a contour gradient wind or the steady natural gradient wind used as an approximation for an actual wind may not be capable of representing the full range of actual wind magnitude.
\end{abstract}

\section{Introduction}

The definition of the gradient wind invoked here is the following paraphrase of Dutton's (1986, p. 311) textbook definition: The gradient wind is a horizontal wind having the same direction as the geostrophic wind but with a magnitude consistent with a balance of three forces: the pressure gradient force, the Coriolis force, and the centrifugal force arising from the curvature of a parcel trajectory. In the usual order of presentation to students, the atmospheric dynamical equations are derived, subjected to scale analysis for large-scale mid- and upper-tropospheric flow, and simplified to explain the concept of the geostrophic wind (e.g., Dutton 1986). The geostrophic wind arises from a balance of the Coriolis and pressure gradient forces. The gradient wind is then introduced as a refinement of the geostrophic wind to

\footnotetext{
* Supplemental information related to this paper is available at the Journals Online website: http://dx.doi.org/10.1175/MWR-D13-00088.s1.

Corresponding author address: Keith F. Brill, NOAA/NWS/ NCEP, NCWCP (W/NP32), Rm. 4630, 5830 University Research Ct., College Park, MD 20740-3818.

E-mail: keith.brill@noaa.gov
}

obtain a better approximation for frictionless large-scale horizontal flow (e.g., Dutton 1986).

From the perspective of the gradient wind as a refinement of the geostrophic wind, it may seem mandatory that the parcel trajectories should depend on the geostrophic streamfunction. Then the trajectory curvature would necessarily result from parcels following the geostrophic streamfunction, even as it changes with time. Yet, using parcel curvature derived from an actual wind does not violate the nominal definition of the gradient wind. It is in consideration of this ambiguity that the author proposes the categorization of gradient wind into two types discussed herein. Haltiner and Martin (1957, p. 183) recognize two types of gradient wind. Their terminology characterizes a horizontal flow as being either "gradient at a point or over an extended path." This article revisits the notion of two ways of obtaining the gradient wind, proposes new terminology, and explores the different types of gradient wind by way of descriptive and quantitative examples.

The concept of the gradient wind is a familiar pedagogical tool found in many textbooks on atmospheric dynamics. The properties of the solutions to the gradient wind quadratic equation have been exhaustively examined (e.g., Fultz 1991). Beyond the classroom in research, the gradient wind is sometimes used as a background or 
reference state in analytic or numerical modeling (e.g., Emanuel 1986; Hodyss and Nolan 2007). In practical applications, the gradient wind may serve as a better estimate of a wind field associated with a mass field than does the geostrophic wind (e.g., Dutton 1986, p. 311; Holton 2004, p. 65).

The goals of the analysis presented here are to draw distinctions among valid methods of computing the gradient wind and to characterize basic differences, both from a computational point of view and how the different gradient wind magnitudes compare to the actual wind as a benchmark. Accomplishing this requires some examination of the fundamental mathematics behind the gradient wind. With reference to the mathematics, the proposed new terminology is offered. In the following, the second section presents a concise background for the mathematical aspects of this analysis, introduces the new terminology, and then derives and discusses a formula for comparing gradient wind and actual wind magnitudes. The third section applies the new terminology, offering both qualitative and quantitative examples of the gradient wind types in comparison to actual winds. The final section presents summarizing material and concluding remarks.

\section{Mathematical analysis}

\section{a. Background}

The gradient wind approximates large-scale horizontal frictionless flow. The horizontal radius of curvature needed to compute the centrifugal acceleration term of the gradient wind equation is the curvature radius of a parcel trajectory. The curvature of a trajectory arises from two effects: the local curvature of the streamlines and the local change with time of the streamlines. Mathematically, this is expressed by Blaton's equation [derived in many atmospheric dynamics textbooks, e.g., Dutton (1986), p. 318]:

$$
K=K_{s}-\frac{1}{V} \frac{\partial \theta}{\partial t}
$$

where $K$ is the horizontal curvature of the trajectory, $V$ is the wind (parcel) speed, $K_{s}$ is the horizontal curvature of the streamline, $\theta$ is the conventional meteorological wind direction measured in radians from north (direction from which the wind is blowing, increasing in the clockwise direction), ${ }^{1}$ and $t$ is the independent variable

\footnotetext{
${ }^{1}$ Some textbooks (e.g., Holton 2004) give the angle in Blaton's equation as the mathematical angular direction, positive in the counterclockwise direction.
}

time. Equation (1) is often expressed in terms of the radii of curvature, which are the reciprocals of the curvature parameters. The second term on the rhs of (1) accounts for the change with time of the streamlines. Curvature is positive (negative) for a cyclonically moving parcel and negative (positive) for the anticyclonic case in the Northern (Southern) Hemisphere. It should be noted that (1) is general and applies to any horizontal wind field.

The infinitesimal displacement of a parcel moving along a curved trajectory occurs in a local horizontal tangent plane and, in the parcel frame of reference, is represented mathematically as an infinitesimally small rotation of a natural coordinate system. The orthogonal natural coordinate system is $(\tau, \eta, \kappa, t)$, for which unit vector $\boldsymbol{\tau}$ is along the horizontal velocity vector $\mathbf{V}$, unit vector $\boldsymbol{\eta}$ is to the left of $\boldsymbol{\tau}$, unit vector $\boldsymbol{\kappa}$ is along the local vertical, and $t$ is the time coordinate. The choice of vertical coordinate determines the formulation expressing the pressure gradient force. In this article, selecting the vertical coordinate to be pressure allows the horizontal pressure gradient force to be expressed as the gradient of the geopotential ( $\phi=g z$, where $g$ is the acceleration of gravity and $z$ is the height of the isobaric surface above mean sea level).

Another basic equation needed is one of two component equations of horizontal frictionless motion in the natural coordinate system. The equation of interest describes the total time rate of change of parcel direction as it moves along the trajectory, but this component equation can be written in terms of the horizontal curvature. The required result is derived concisely in Holton (2004, section 3.2.1). Rewriting Holton's (2004) Eq. (3.10) (p. 61) in terms of the coordinate system described above with the radius of curvature replaced by its reciprocal, the curvature, and rearranging yields

$$
K V^{2}+f V+\frac{\partial \phi}{\partial \eta}=0
$$

where $f$ is the Coriolis parameter.

Equation (2) is valid in either the Northern or Southern Hemisphere. In crossing the equator from one hemisphere to the other, both $K$ and $f$ change algebraic signs. The geopotential gradient term also changes sign for a symmetrical configuration reflected across the equator in the opposite hemisphere. Consider a midlatitude geopotential trough in the Northern Hemisphere with westerly flow at the base and $\boldsymbol{\eta}$ directed to the left of the vector wind in the poleward direction giving $\partial \phi / \partial \eta<0$. For a similar trough in the Southern Hemisphere, there is also westerly flow at the base of the trough with $\boldsymbol{\eta}$ directed to the left of the vector wind, but in the equatorward 
direction, giving $\partial \phi / \partial \eta>0$. Thus, applying (2) to an identical flow pattern on the opposite side of the equator is like multiplying both sides of that equation by -1 . Finally, regardless of hemisphere, $K f>0$ defines cyclonic flow, and $K f<0$ defines anticyclonic flow [following Holton (2004), p. 67].

The gradient wind equation is obtained from (2) by replacing $K$ with the gradient wind curvature $K_{\mathrm{gr}}$ and replacing $\partial \phi / \partial \eta$ with $-f V_{g}$, where $V_{g}$ is the magnitude of the geostrophic wind:

$$
K_{\mathrm{gr}} V_{\mathrm{gr}}^{2}+f V_{\mathrm{gr}}-f V_{g}=0,
$$

where $V_{\mathrm{gr}}$ is the magnitude of the gradient wind. [The gradient wind has the same direction as the geostrophic wind, hence, the replacement of the geopotential gradient term in (2) with the equivalent geostrophic wind term in deriving (3).] If the actual curvature of a real wind trajectory is known and the direction differs only slightly from geostrophic, then by (2) the gradient wind magnitude will be very nearly equal to that of the wind.

With the gradient wind curvature $K_{\text {gr given by applying }}$ (1) to a geostrophic or an actual wind field, the gradient wind equation is a quadratic equation in the unknown variable $V_{\mathrm{gr}}$. Substituting Blaton's equation directly into the gradient wind equation yields a general form for the gradient wind equation (Dutton 1986, p. 318):

$$
K_{s} V_{\mathrm{gr}}^{2}+\left(f-\frac{\partial \theta_{s}}{\partial t}\right) V_{\mathrm{gr}}-f V_{g}=0 .
$$

Solving (3) or (4) is covered in many textbooks (e.g., Haltiner and Martin 1957, p. 189; Holton 2004, 65-67) and is assumed to be familiar to the reader; so, only a brief summary of the solutions is rendered in this article. For cyclonic curvature, the square root term in the solution is always real valued, but only one of the two solutions gives a positive gradient wind magnitude. For anticyclonic curvature, the square root term may be imaginary, in which case a gradient wind magnitude cannot be computed. If the square root is real valued for anticyclonic curvature, then both the positive and the negative values of the square root result in positive solutions. The lesser of the two solutions for anticyclonic curvature is called the "normal" solution. The greater of the two solutions for anticyclonic curvature is termed the "anomalous" solution. The anomalous solution is rarely appropriate (Haltiner and Martin 1957, p. 192), but winds matching the anomalous solution have been documented (Mogil and Holle 1972).

Actual wind data are needed to justify the choice of the anomalous solution as exemplified in the approach of Mogil and Holle (1972). This is a point that deserves particular emphasis. For anticyclonic curvature, both the normal and anomalous solutions for the gradient wind magnitude exceed the geostrophic wind (Holton 2004, p. 67). Therefore, there is no basis for determining whether or not to choose the anomalous solution without looking beyond the geostrophic wind to the actual wind. If the anomalous gradient wind magnitude is a better match to the actual wind speed, then it is appropriate to choose the anomalous solution.

The streamline curvature $K_{s}$ and streamline time tendency as measured by $\partial \theta_{s} / \partial t$ in (4) may be that of the geostrophic wind or the actual wind. While the direction of the gradient wind is clearly defined to be identical to that of the geostrophic wind, the magnitude of the gradient wind, computed using (4), is dependent on the values of $K_{s}$ and $\partial \theta_{s} / \partial t$, which may be obtained from either the geostrophic wind or the actual wind, if the latter is available. From the standpoint of (3), $K_{\mathrm{gr}}$ may be the curvature of a parcel trajectory following the geostrophic wind or following the actual wind.

The fact that the choice between two wind fields may exist for the curvature calculation provides the basis for the proposed terminology, contour versus natural gradient winds. If the streamlines of the geostrophic wind are used in (4), the result is a contour gradient wind. If the streamlines of the actual wind are used in (4), the result is a natural gradient wind. If $\partial \theta_{s} / \partial t \equiv 0$ in (4), the gradient wind of either type is steady; otherwise, it is nonsteady. Haltiner and Martin (1957, p. 183) describe "extended gradient flow" and flow that is "gradient at a point," which correspond to the author's contour and natural gradient winds, respectively. The determination of this correspondence is based in part on Haltiner and Martin's elaboration: "For extended gradient flow, the wind is parallel to the isobars along the entire trajectory." The "point" denomination arises from the fact that if the actual wind at a given point location has the same direction as the geostrophic wind, then using the curvature of the actual wind parcel trajectory in the gradient wind equation gives a gradient wind magnitude equal to the actual wind speed. Thus, the actual wind is "gradient" at such a point. The terminology of Haltiner and Martin (1957) is awkward, and perhaps confusing, because all gradient wind calculation methods can be carried out at a point and all require the curvature of some sort of trajectory (i.e., an extended path). In any case, to the author's knowledge, the terminology of Haltiner and Martin (1957) is not in common use. However, the new terms harken back to Haltiner and Martin's (1957) descriptive nomenclature. The "extended path" suggests the term "contour." The "at a point" gradient wind suggests the term "natural" because any observation of true gradient balance in nature requires use of actual wind data for its determination. 
TABLE 1. Relative values for $K^{\prime}$ and $K_{\mathrm{gr}}^{\prime}$ corresponding to supergradient conditions for the steady contour, nonsteady contour, or steady natural gradient winds. The tabulated results assume that the last two terms of (5) enclosed by parentheses are not of sufficient consequence to cause subgradient conditions. The table entries also assume that a gradient wind solution is possible.

\begin{tabular}{cccccc}
\hline \hline Row & $K^{\prime}$ & $K_{\mathrm{gr}}^{\prime}$ & Actual wind curvature & Gradient wind curvature & Condition for $V-V_{\mathrm{gr}}>0$ \\
\hline 1 & $<0$ & $<0$ & Anticyclonic & Anticyclonic & $K^{\prime} \leq K_{\mathrm{gr}}^{\prime}$ is sufficient \\
2 & $<0$ & $>0$ & Anticyclonic & Cyclonic & No restriction \\
3 & $>0$ & $<0$ & Cyclonic & Anticyclonic & Never possible \\
4 & $>0$ & $>0$ & Cyclonic & Cyclonic & $K^{\prime}<K_{\mathrm{gr}}^{\prime}$ is necessary \\
\hline
\end{tabular}

\section{b. Assessment of supergradient winds}

Each type of gradient wind has a different ability to estimate the wind speed compared to that of the actual wind. The mathematics of section $2 \mathrm{a}$ can be used to address the question as to the possibility for supergradient conditions, for which the actual wind speed exceeds the gradient wind speed. One way to assess the supergradient possibility is to subtract the gradient wind equation (3) from (2) and solve for $V-V_{\text {gr }}$ yielding

$$
V-V_{\mathrm{gr}}=V_{\mathrm{gr}}^{2} K_{\mathrm{gr}}^{\prime}-V^{2} K^{\prime}-\left(V_{g}+\frac{1}{f} \frac{\partial \phi}{\partial \eta}\right)
$$

where $K_{\mathrm{gr}}^{\prime}=K_{\mathrm{gr}} / f$ and $K^{\prime}=K / f$. Regardless of hemisphere, $K_{\mathrm{gr}}^{\prime}>0$ indicates cyclonic curvature; whereas, $K_{\mathrm{gr}}^{\prime}<0$ indicates anticyclonic curvature. The same applies to $K^{\prime}$. The indications of the character of curvature for $K_{\mathrm{gr}}^{\prime}$ and $K^{\prime}$ are consistent with definitions for cyclonic and anticyclonic curvature given in the discussion following (2) above. If the wind and geostrophic wind directions are identical, the last two terms grouped within parentheses on the rhs of (5) exactly sum to zero, because $f V_{g}=-\partial \phi / \partial \eta$ in that case. If the actual wind deviates from the geostrophic direction (cross-contour flow), then $\partial \phi / \partial \eta$ is decreased in magnitude because $\boldsymbol{\eta}$ is not pointing in the direction of greatest change of $\phi$. For cross-contour flow, $(1 / f)(\partial \phi / \partial \eta)$, which is intrinsically negative, cannot take away the full value of $V_{g}{ }^{2}$ Thus, for cross-contour flow by the actual wind, these last two parenthetically enclosed terms of (5) evaluate to a positive number rather than zero. This positive value is subtracted, diminishing any supergradient wind effect that may arise from the first two terms on the rhs of (5).

The effect of the first two terms on the rhs of (5) is examined in the following. Table 1 applies for steady contour, nonsteady contour, or steady natural gradient winds. Nonsteady natural gradient winds are treated as a separate case below. The last column of Table 1 states

\footnotetext{
${ }^{2}$ This article assumes all flows are baric with positive values of the geostrophic wind (Holton 2004, p. 67).
}

the condition that does not contradict $V>V_{\mathrm{gr}}$ for given curvatures of the actual and gradient winds. For example, considering the first row of Table 1 , both $K^{\prime}$ and $K_{\mathrm{gr}}^{\prime}$ are intrinsically negative, making the $V^{2}$ term of (5) positive and the $V_{\mathrm{gr}}^{2}$ term negative. But, since $V^{2}$ is larger than $V_{\mathrm{gr}}^{2}$ when $V>V_{\mathrm{gr}}$, it is sufficient that $\left|K^{\prime}\right| \geq\left|K_{\mathrm{gr}}^{\prime}\right|$ for $\left(V^{2}\left|K^{\prime}\right|-V_{\mathrm{gr}}^{2}\left|K_{\mathrm{gr}}^{\prime}\right|\right)>0$. Thus, it follows that if both $K_{\mathrm{gr}}^{\prime}$ and $K^{\prime}$ are intrinsically negative, it is sufficient that $K^{\prime} \leq K_{\mathrm{gr}}^{\prime}$ to support the supergradient condition. Similar reasoning leads to the conditions given in the last column of Table 1 for the remaining rows. According to the first row of Table 1, if both the actual wind and the gradient wind are characterized by anticyclonic curvature, then the actual wind must be at least as anticyclonically curved as the gradient wind for super gradient conditions to prevail. By row 2 of Table 1, if the actual curvature is anticyclonic and the gradient wind curvature is cyclonic, supergradient conditions are possible without restriction [excepting the effect of the last two terms of (5)]. On the other hand, if the actual wind is cyclonic and the gradient wind is anticyclonic, supergradient conditions cannot occur according to row 3 of Table 1 . Finally, with reference to row 4 of Table 1 , if both the actual wind and the gradient wind are cyclonic, then the actual wind must be less cyclonically curved for the flow to be supergradient. Table 1 shows that the steady contour, the nonsteady contour, or the steady natural gradient wind may not represent the maximum potential magnitude for the actual wind.

In the case of the nonsteady natural gradient wind, whose curvature is the same as that of the actual wind by definition, $K_{\mathrm{gr}}^{\prime}=K^{\prime}$, and (5) becomes

$$
V-V_{\mathrm{gr}}=\left(V_{\mathrm{gr}}-V\right)\left(V_{\mathrm{gr}}+V\right) K^{\prime}-\left(V_{g}+\frac{1}{f} \frac{\partial \phi}{\partial \eta}\right) .
$$

For cyclonic curvature $\left(K^{\prime}>0\right)$, assuming $\left(V-V_{\text {gr }}\right)>0$ leads to an impossibility for (6) because the rhs is less than zero under that assumption. For anticyclonic curvature, $\left(V-V_{\mathrm{gr}}\right)>0$ is allowed by (6), but only for impossible values of $K^{\prime}$ that yield an imaginary square root in the solution to the gradient wind equation. This is demonstrated in the appendix. Therefore, the normal-solution 


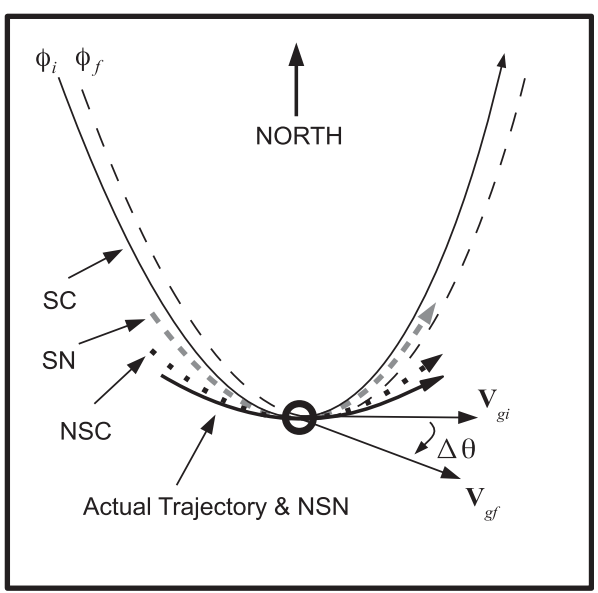

FIG. 1. Idealized tangent plane schematic (Northern Hemisphere) shows four trajectories passing through the point circumscribed by the thick circle: the steady contour gradient wind trajectory (SC, thin solid with arrow), the nonsteady contour gradient wind trajectory (NSC, thick dotted with arrow), the steady natural gradient wind ( $\mathrm{SN}$, light dashed with arrow), and the nonsteady natural gradient wind having the actual wind trajectory (NSN, thick solid with arrow). The initial and final positions of a contour of the geostrophic streamfunction, $\phi$ for a small change in time are shown and indicated by subscripts $i$ and $f$, respectively. The local change in direction of the geostrophic wind at the circumscribed point is depicted by the initial and final geostrophic wind vectors, $\mathbf{V}_{\text {gi }}$ and $\mathbf{V}_{\text {gf }}$, respectively, along with the angular change $\Delta \theta$.

nonsteady natural gradient wind speed stands as an upper bound for the actual wind speed. This agrees with a similar conclusion stated on p. 192 of Haltiner and Martin (1957) for the at a point gradient wind.

\section{Demonstrating the new terminology}

\section{a. Schematic illustration}

Figure 1 presents schematically a collection of possible trajectories occurring for different assumed conditions for contour or natural gradient winds. Figure 1 depicts an idealized large-scale mid- or upper-tropospheric trough occurring well away from the equator in the Northern Hemisphere. The trajectory assessment in Fig. 1 applies at the instant the parcel passes through the identified point circumscribed by the thick circle. If steady flow conditions are assumed for the contour gradient wind, then only the first term on the rhs of (1) imparts curvature and the parcel follows the contour of the geostrophic streamfunction. This steady contour (SC) gradient wind trajectory is identified by SC in Fig. 1 and is coincident with the geopotential contour labeled $\phi_{i}$. The nonsteady contour gradient wind trajectory (NSC in Fig. 1) has less cyclonic curvature than the SC because both terms on the rhs of (1) are contributing to the curvature. For the progressive translation of the trough from the position of contour $\phi_{i}$ to contour $\phi_{f}$ depicted in Fig. 1, the direction from north of the geostrophic wind increases at the identified point; so, $\Delta \theta>0$, and $\partial \theta / \partial t$ in (1) is positive $\left[\partial \theta_{s} / \partial t>0\right.$ in (4)] causing the second term of (1) to subtract from the first, thereby decreasing the magnitude of cyclonic curvature. If the translation were retrogressive, the magnitude of cyclonic curvature would increase.

The steady natural (SN) gradient wind is drawn somewhat arbitrarily and labeled SN in Fig. 1. If the large-scale mid- or upper-tropospheric trough depicted in Fig. 1 is well away from the equator, then "the geostrophic wind approximates the true horizontal velocity to within $10 \%-$ $15 \%$ " (Holton 2004, p. 40). For this reason the SN trajectory is depicted so that the instantaneous streamline of the actual wind is not much different from the instantaneous geostrophic streamfunction $\phi_{i}$. Finally, the trajectory of the real wind, which is also the trajectory of the nonsteady natural gradient wind (NSN in Fig. 1), has curvature determined by progressively translating the SN streamline in the same way as described above for the translation of the SC streamline, which resulted in less cyclonic curvature for the NSC trajectory. Likewise, a similar argument applied for translating the SN streamline results in a decrease in the magnitude of cyclonic curvature for the NSN trajectory as depicted in Fig. 1.

Figure 1 is intended primarily to illustrate different trajectory paths possible for steady and nonsteady contour and natural gradient winds in an idealized schematic form. Figure 1 also serves to demonstrate the influence of Blaton's equation for the nonsteady cases. Although Fig. 1 is not a quantitative depiction, the relative magnitudes of the gradient wind at the identified point can be inferred by examining the curvature of the trajectories. In order of SC, SN, NSC, and NSN, the curvatures become less cyclonic, and the centrifugal force (directed to the right in Fig. 1) in the gradient wind balance becomes less for each successive trajectory in that order. Therefore, the gradient wind magnitude must increase in the progression of SC, SN, NSC, and NSN because an increased Coriolis force (also directed to the right) is needed to balance the same pressure gradient force (directed to the left). The sequence of curvature change depicted in Fig. 1 applies only to the particular case shown and will vary in general. For example, if the motion were retrogressive in Fig. 1 as mentioned earlier, the nonsteady gradient wind trajectories would become more cyclonically curved and the corresponding gradient wind magnitudes would be less.

Although Fig. 1 applies to the Northern Hemisphere, a simple exercise can transform it into a Southern Hemisphere depiction: place an inverted display of Fig. 1 facing a mirror and view the reflected image. The arrow labeled 
"NORTH" is pointing south. The translation of the trough is still west to east (from left to right). The turning of the geostrophic wind vector associated with the translation of the trough is counterclockwise rather than clockwise. In the Southern Hemisphere, cyclonic curvature is negative; so, with $\Delta \theta<0$ and $\partial \theta / \partial t$ in (1) negative $\left[\partial \theta_{s} / \partial t<0\right.$ in (4)], the second term of (1) adds to the first, thereby decreasing the magnitude of cyclonic curvature as in the Northern Hemisphere. And so it follows, all of the conclusions reached above for the Northern Hemisphere are the same in the Southern Hemisphere.

\section{b. Example gradient wind calculations}

To show quantitative examples, the four species of gradient wind are computed using the solution to (3) or (4) and displayed in Fig. 2 as differences between the actual wind magnitude and the gradient wind magnitude. The calculations for Fig. 2 use forecast fields from the National Centers for Environmental Prediction (NCEP) Global Forecast System (GFS). The forecast projections are 24 and $27 \mathrm{~h}$ corresponding to forecasts of upper-tropospheric flow having good agreement to verifying analyses. The calculations are carried out using the average of model output fields at 24 and $27 \mathrm{~h}$. Specific date information is given in the Fig. 2 caption. Time tendency calculations needed for the nonsteady contour gradient winds are based on the difference between the 27 - and 24 -h forecast fields. The GFS data are at $1^{\circ} \times 1^{\circ}$ horizontal resolution on a simple cylindrical projection. Since the gradient wind applies to large-scale flows and to ensure aesthetically appealing displays, the data are filtered (the $e$-folding wavelength is seven grid spaces for a filter having a Gaussian distribution of weights) so that the numerical values of gradients are more typical of those obtained for coarser spatial resolution with larger separation of grid points. The General Meteorological Package (GEMPAK) described by desJardins et al. (1991) was invoked for gridded data computation and graphical displays. The GEMPAK programs and parameter settings used for the computations and graphics are provided in a UNIX shell context in the supplemental material.

The streamline curvature for applying (3) or (4) is computed from the expression for the vertical component of relative vorticity $\zeta$ in the natural coordinate framework (Dutton 1986, p. 337):

$$
\zeta=V K_{s}-\frac{\partial V}{\partial \eta} \rightarrow K_{s}=\frac{1}{V}\left(\zeta+\frac{\partial V}{\partial \eta}\right) .
$$

In the coordinate system on the map projection plane of the GFS output, the $\partial V / \partial \eta$ term is evaluated as a directional derivative of the wind magnitude along a unit vector in the $\boldsymbol{\kappa} \times \mathbf{V}$ direction. For contour gradient winds, the vector wind for the directional derivative, the wind magnitude, and the vorticity in (7) are from the geostrophic wind field, whereas, for the steady natural gradient winds, these three are from the actual wind field. For the nonsteady natural gradient wind, the trajectory curvature is obtained by solving (2) for $K$ as $K_{\mathrm{gr}}$, yielding

$$
K_{\mathrm{gr}}=-\frac{1}{V^{2}}\left(f V+\frac{\partial \phi}{\partial \eta}\right) .
$$

For (8), $\partial \phi / \partial \eta$ is evaluated as a directional derivative in the $\boldsymbol{\kappa} \times \mathbf{V}$ direction, where $\mathbf{V}$ is the actual wind. The $V$ in (8) is the actual wind magnitude. With $K_{\mathrm{gr}}$ determined by (8), the gradient wind equation in the form of (3) is solved to get the nonsteady natural gradient wind magnitude. For the nonsteady contour gradient wind, (4) must be used, and the wind direction tendency term in (4) is computed using a 3-h finite difference of the geostrophic wind direction.

Although the solution to the gradient wind equation is well behaved for significant cyclonic curvature (e.g., tropical cyclones), it is not as easily computed when the flow regime manifests both cyclonic and anticyclonic curvature, as is typically the case for large-scale midlatitude flows. In the transition from cyclonic to anticyclonic curvature, the curvature value passes through zero. For nearzero streamline curvature $\left(-10^{-8}<K_{s}<10^{-8} \mathrm{~m}^{-1}\right)$, the linear solution after neglecting the quadratic term of (4) is used in creating Fig. 2:

$$
V_{\mathrm{gr}}=f\left(f-\frac{\partial \theta_{s}}{\partial t}\right)^{-1} V_{g} .
$$

Other challenges for the computation of the gradient wind magnitude are the occurrence of the imaginary square root and the existence of the anomalous solution for anticyclonic curvature (Haltiner and Martin 1957, p. 189). The imaginary square root results in missing values (areas not shaded in Fig. 2), and the anomalous case is avoided by computing only the normal solution. Mogil and Holle (1972) present example cases for the anomalous solution calculated using the nonsteady natural gradient wind.

The other practical aspects of the calculations for Fig. 2 are the time averaging and smoothing mentioned previously in discussing the source of data. Both help to prevent small-scale spatial fluctuations in curvature, especially in the regions of transition from cyclonic to anticyclonic curvature or vice versa. Smoothing reduces noisy small-scale variations in the magnitude of the geostrophic wind. The time averaging and smoothing act to eliminate any spurious influences of gravity waves associated with topography or geostrophic adjustment (Holton 2004) occurring on a scale smaller than that typically associated with midlatitude gradient wind balance. 


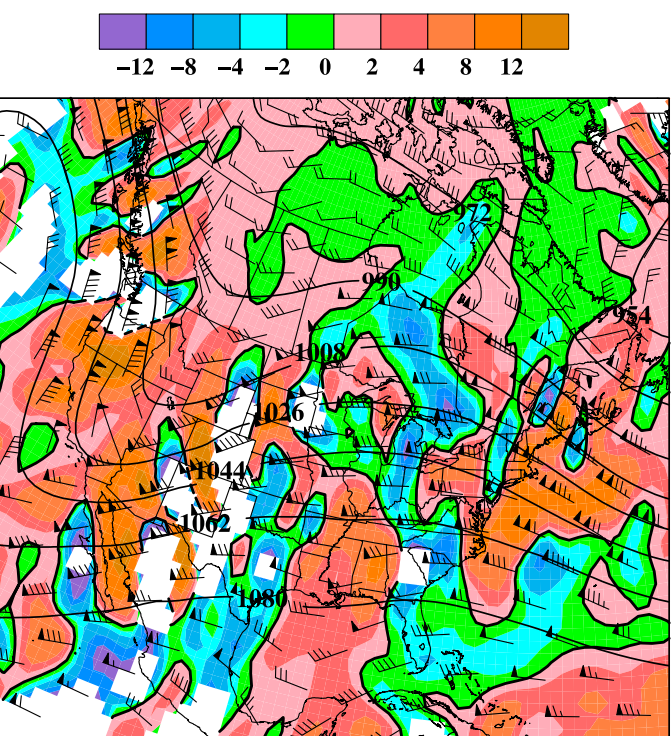

a) STEADY CONTOUR

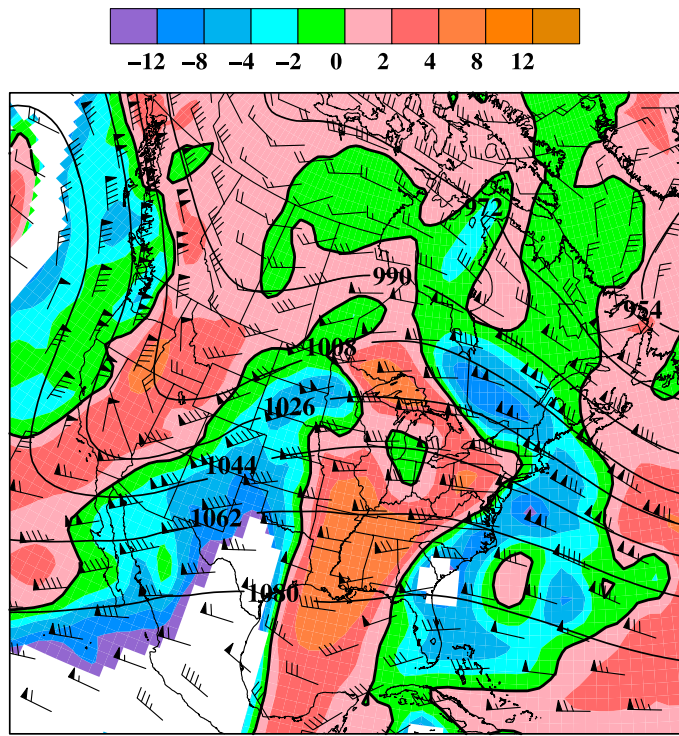

c) STEADY NATURAL

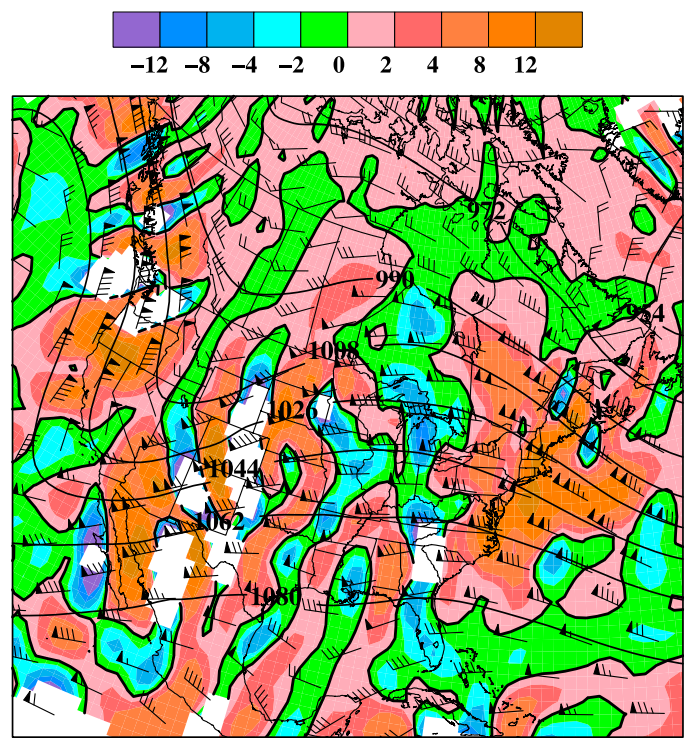

b) NON-STEADY CONTOUR

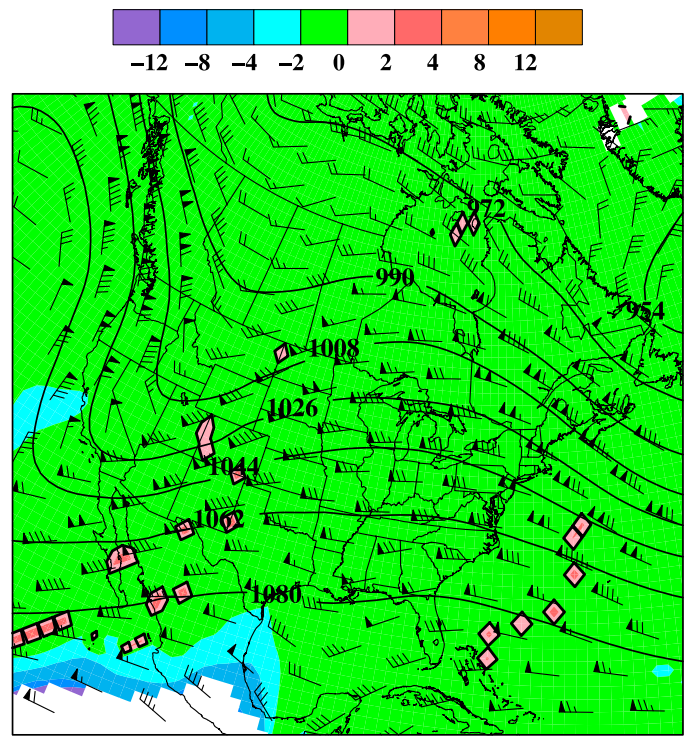

d) NON-STEADY NATURAL

FIG. 2. Differences between actual wind magnitude and that of the gradient wind $\left(V-V_{\mathrm{gr}}, \mathrm{m} \mathrm{s}^{-1}\right.$, shaded, color bar at top) are shown for (a) steady contour, (b) nonsteady contour, (c) steady natural, and (d) nonsteady natural gradient winds at the 250 -hPa level. The data are $1^{\circ} \times 1^{\circ}$ latitude-longitude filtered GFS forecast grids, averages of the 24- and 27-h forecasts from initial time 1200 UTC 27 Jan 2013, with the valid time at the interval midpoint (1330 UTC 28 Jan 2013). Averaged height contours (dam, thin, solid) are shown. Averaged forecast actual winds are shown by wind barbs $\left(\mathrm{m} \mathrm{s}^{-1}\right.$, half barb is 2.5 , full barb is 5 , flag is 25$)$. Nonshaded areas are where the gradient wind calculation fails. The zero contour for the differences is drawn as a thick solid line.

Figure 2 is designed to reveal with little more than a glance that the various kinds of gradient wind are in fact different as evidenced by the varying color shaded patterns of supergradient (pink to brown) and subgradient (green to purple) flow. The gradient wind magnitude itself is obtained by subtracting the shaded difference value from the actual wind magnitude shown by the wind barbs. Super- and subgradient wind speeds are evident for both steady and nonsteady contour gradient winds as well as for the steady natural gradient wind. Only subgradient flow is found for the nonsteady natural gradient wind, except for small isolated areas where the linear solution using (9) is required. Similar small isolated areas requiring (9) also exist for the other 


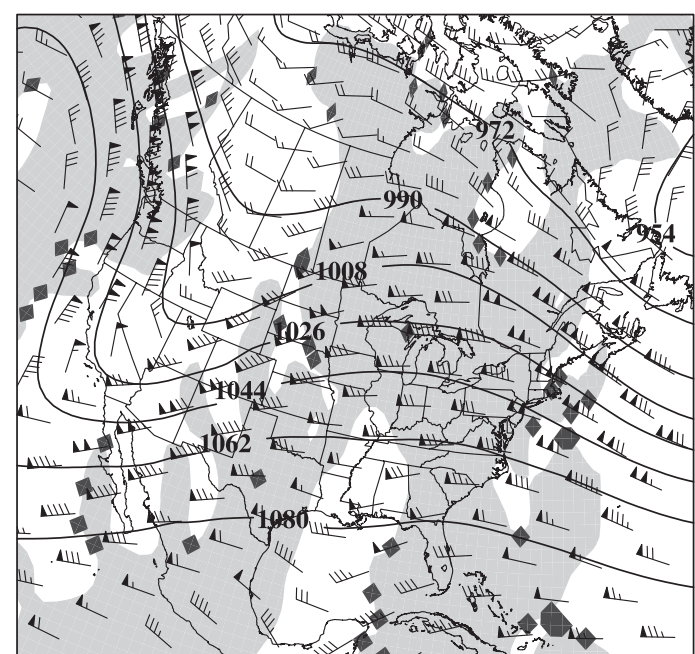

a) STEADY CONTOUR

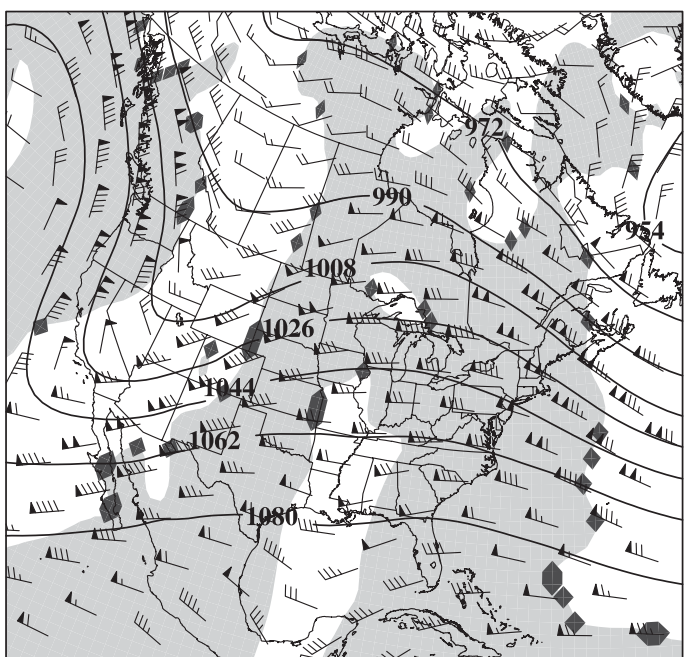

c) STEADY NATURAL

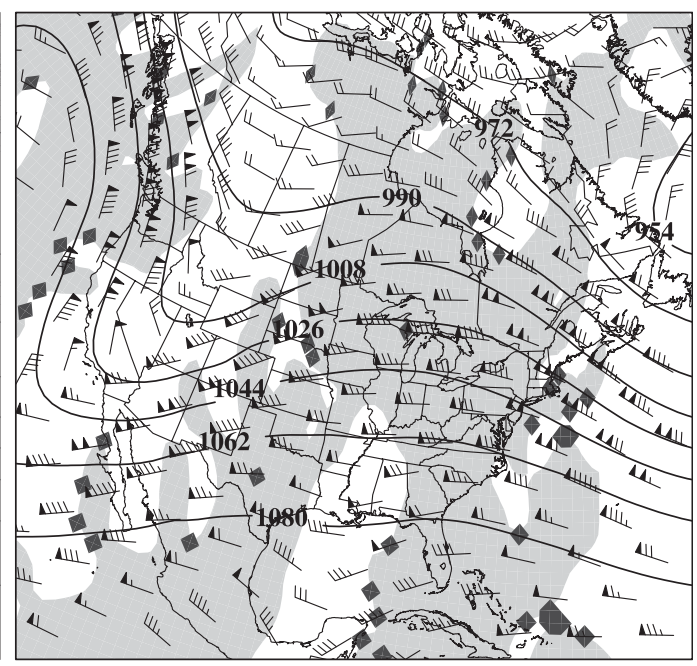

b) NON-STEADY CONTOUR

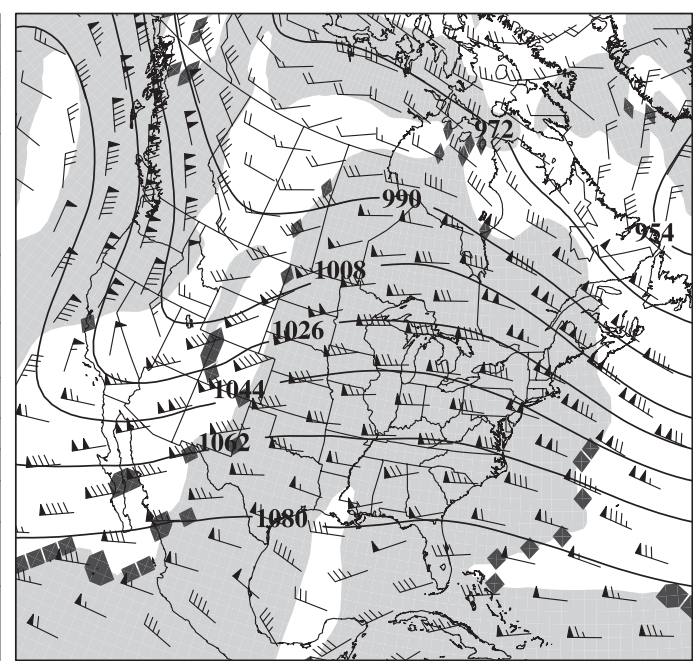

d) NON-STEADY NATURAL

FIG. 3. Character of curvature (anticyclonic light shaded, cyclonic not shaded) for (a) steady contour, (b) nonsteady contour, (c) steady natural, and (d) nonsteady natural gradient winds at the 250-hPa level, corresponding to the gradient winds depicted in Fig. 2. Dark shaded areas denote regions of near zero curvature requiring the application of (9) in the calculation of gradient winds displayed in Fig. 2.

types of gradient wind and are shown as scattered dark shaded regions in Fig. 3.

In Fig. 3, the regions of anticyclonic curvature are lightly shaded in gray. Figure 3 is provided to facilitate a deeper discussion of the patterns seen in Fig. 2. The curvature of the actual wind is shown in Fig. 3d. In general, there is agreement among the different curvature calculations for cyclonic curvature (nonshaded areas in Fig. 3) associated with the trough over the western continental United States (CONUS). The trough off the eastern coast of the CONUS is also an area of general agreement on cyclonic curvature. Areas of anticyclonic curvature over the northwestern CONUS and over the eastern CONUS and southeastern Canada are also generally evident in all four panels of Fig. 3. However, an area of disagreement between the character of curvature of the nonsteady natural gradient wind (also the curvature of the actual wind) in Fig. 3d and that of the other gradient winds is apparent over the lower (southern) Mississippi Valley (LMV). The curvature of the actual wind (Fig. 3d) is anticyclonic over the LMV region, except for a narrow strip of cyclonic curvature extending from southern Louisiana southward into the central Gulf of Mexico where actual wind barbs suggest a shortwave trough. Figures $3 a-c$ show cyclonic curvature over the LMV. By the second row of Table 1, the LMV area 
TABLE 2. Example Lagrangian gradient wind calculations for a 3-h segment of the cold conveyor belt trajectory from Table 3 of Whitaker et al. (1988). The successive positions numbered in the first column are the ending points for each subsegment and are separated by $1 \mathrm{~h}$. The entries for the vector wind (parcel velocity) $\mathbf{V}$ and vector geostrophic wind $\mathbf{V}_{g}$ are given in the form of direction/ speed, where the direction is the conventional direction from which the wind is blowing measured in degrees from north and the speed is in $\mathrm{m} \mathrm{s}^{-1}$. The parcel curvature $K_{p}$ is $\mathrm{m}^{-1}$. The gradient wind speed $V_{\mathrm{gr}}$ is given in $\mathrm{m} \mathrm{s}^{-1}$. Latitude and longitude are expressed in degrees, with longitude negative west of the Greenwich meridian. The average geostrophic wind speed for each hourly segment is used in the gradient wind computation.

\begin{tabular}{lcccccl}
\hline \hline Position & $\begin{array}{c}\text { Lat } \\
\left({ }^{\circ} \mathrm{N}\right)\end{array}$ & $\begin{array}{c}\text { Lon } \\
\left({ }^{\circ} \mathrm{W}\right)\end{array}$ & $\mathbf{V}$ & $\mathbf{V}_{g}$ & $K_{\mathrm{gr}}=K_{p}$ & $V_{\mathrm{gr}}$ \\
\hline Initial & 34.1 & -70.9 & $132 / 20$ & $176 / 23$ & & \\
1 & 34.6 & -71.5 & $134 / 24$ & $175 / 37$ & $-4.46 \times 10^{7}$ & 38 \\
2 & 35.3 & -72.4 & $133 / 30$ & $186 / 47$ & $1.54 \times 10^{-7}$ & 39 \\
3 & 36.1 & -73.3 & $147 / 37$ & $248 / 25$ & $-2.03 \times 10^{6}$ & Complex \\
\hline
\end{tabular}

should be quite susceptible to supergradient winds for all species of gradient winds except the nonsteady natural gradient wind because the actual wind curvature is anticyclonic and the gradient wind curvature is cyclonic. The presence of supergradient winds over the LMV is confirmed in Figs. 2a-c.

The example gradient wind calculations presented thus far have been based on Eulerian data from a numerical weather prediction (NWP) model. In some cases Lagrangian data are available from NWP models, such as the high temporal resolution trajectory data used by Uccellini et al. (1987) and Whitaker et al. (1988). For trajectories, parcel positions are determined using frequent model or analysis output, and the actual wind and geostrophic wind speeds and directions are interpolated to the parcel locations. The calculation of the curvature of a trajectory requires only the finite difference form of Dutton's (1986, p. 317) Eq. (11):

$$
K_{p}=-\frac{\Delta \theta}{\Delta s},
$$

where $K_{p}$ is the curvature of the parcel path and $\Delta \theta$ is the change of the wind direction for the parcel as it moves a distance $\Delta s$ from one discrete point location to the next. Since the trajectory curvature is based on actual wind data that are changing in time, Lagrangian-derived gradient winds are natural gradient winds (nonsteady in general, but possibly steady) that can be computed by solving (3) with $K_{\mathrm{gr}}=K_{p}$. Whether or not gradient winds calculated from Lagrangian data are steady depends on the wind field in which the parcel is embedded.

Table 2 displays trajectory-based gradient wind calculations utilizing model data tabulated by Whitaker et al.
(1988). Although the underlying Lagrangian output for the Whitaker et al. (1988) trajectories had 15-min temporal resolution, the published tabulated data are less frequent (hourly in Table 2), and the numerical values have limited arithmetic precision (e.g., positions given only to the nearest tenth of a degree of latitude and longitude). Given the relative imprecision of the data, the distance calculation used for Table 2 is the Cartesian distance estimate of the great circle arc length from the initial to the final parcel position:

$$
\Delta s \approx R_{e}\left[\Delta \lambda^{2} \cos (\bar{\varphi})^{2}+\Delta \varphi^{2}\right]^{1 / 2},
$$

where $R_{e}$ is Earth's radius (taken to be $6371 \mathrm{~km}$ ), $\Delta \lambda$ is the change in longitude for the parcel displacement, $\Delta \varphi$ is the change in latitude, and $\bar{\varphi}$ is the average latitude over the parcel displacement. The trajectory data selected for Table 2 are taken from Table 3 of Whitaker et al. (1988) and are chosen for a variably curved portion of the cold conveyor belt (described originally by Carlson 1980) trajectory for which the vertical displacement in the pressure coordinate is only $17 \mathrm{hPa}$ in $3 \mathrm{~h}$ thereby approximately satisfying the assumption of horizontal motion for the gradient wind. The first 1-h segment (initial position to position 1 in Table 2 ) has anticyclonic curvature, but the gradient wind solution is real valued. The cyclonic curvature of the second 1-h segment ensures a nonimaginary square root in the gradient wind magnitude solution. The third segment is anticyclonic once again, but this time the solution is complex; so, the gradient wind cannot be computed for the third hour. The Table 2 caption describes the contents of the columns and gives other details. The actual parcel speeds for segments 1 and 2 in Table 2 are subgradient as would be expected for the nonsteady natural gradient wind. There is noticeable cross-contour flow evidenced in Table 2 by the differences between the direction of the parcel and that of the geostrophic wind at all positions of the parcel at which the gradient wind exists. The crosscontour parcel direction contributes to the amount of difference of the parcel speed from the gradient wind speed in accordance with (5), because the last two terms of (5) are making an important contribution.

\section{Summary and conclusions}

While the gradient wind is precisely defined with respect to its direction, the magnitude of the gradient wind is not so precisely defined owing to its dependence on the curvature of a parcel trajectory, which may follow the geostrophic wind or the actual wind. Thus, the nominal definition of the gradient wind leaves ambiguous the method of computing the requisite radius of curvature. 
TABLE 3. Summary of types of gradient wind calculations with advantages and disadvantages inferred from this article.

\begin{tabular}{|c|c|c|c|c|c|}
\hline Type & Subtype & $\begin{array}{l}\text { Curvature } \\
\text { related calculations }\end{array}$ & $\begin{array}{c}\text { Gradient } \\
\text { wind equation }\end{array}$ & Advantages & Disadvantages \\
\hline Contour & Steady & $\begin{array}{l}\text { Apply (7) using } \\
\text { geostrophic wind }\end{array}$ & (3) with $K_{\mathrm{gr}}=K_{s}$ & Computed easily & $\begin{array}{l}\text { 1. Either over or underestimates } \\
\text { speed compared to actual wind } \\
\text { 2. Cannot justify anomalous } \\
\text { solution }\end{array}$ \\
\hline Contour & Nonsteady & $\begin{array}{l}\text { Apply (7) using } \\
\text { geostrophic wind; } \\
\text { compute time tendency } \\
\text { of geostrophic } \\
\text { wind direction }\end{array}$ & (4) & $\begin{array}{l}\text { May improve wind speed } \\
\text { estimate compared } \\
\text { to steady contour } \\
\text { gradient wind }\end{array}$ & $\begin{array}{l}\text { 1. Difficult to compute- } \\
\text { Requires time tendency } \\
\text { 2. Either over or underestimates } \\
\text { speed } \\
\text { 3. Cannot justify anomalous } \\
\text { solution }\end{array}$ \\
\hline Natural & Steady & $\begin{array}{l}\text { Apply (7) using } \\
\text { actual wind }\end{array}$ & (3) with $K_{\mathrm{gr}}=K_{s}$ & $\begin{array}{l}\text { 1. Computed easily } \\
\text { 2. Anomalous solution } \\
\text { justifiable }\end{array}$ & $\begin{array}{l}\text { 1. Requires both } \mathbf{V} \text { and } \mathbf{V}_{g} \\
\text { 2. Either over- or underestimates } \\
\text { speed }\end{array}$ \\
\hline Natural & Nonsteady & $\begin{array}{l}\text { Apply (8) using } \\
\text { actual wind }\end{array}$ & (3) & $\begin{array}{l}\text { 1. Computed easily } \\
\text { 2. Reveals degree of actual } \\
\text { gradient balance } \\
\text { 3. Anomalous solution } \\
\text { justifiable }\end{array}$ & $\begin{array}{l}\text { 1. Requires both } \mathbf{V} \text { and } \mathbf{V}_{g} \\
\text { 2. Overestimates speed }\end{array}$ \\
\hline Natural & $\begin{array}{l}\text { Either; } \\
\text { depends } \\
\text { on flow }\end{array}$ & Apply (10) & (3) with $K_{\mathrm{gr}}=K_{p}$ & $\begin{array}{l}\text { 1. Provides the only option } \\
\text { for parcel trajectory data } \\
\text { 2. Reveals degree of actual } \\
\text { gradient balance } \\
\text { 3. Anomalous solution } \\
\text { justifiable }\end{array}$ & $\begin{array}{l}\text { 1. Requires high-resolution } \\
\text { Lagrangian data } \\
\text { 2. Overestimates speed }\end{array}$ \\
\hline
\end{tabular}

This article defines new terminology for two types of gradient wind based upon how the parcel curvature is computed: contour gradient winds are computed using the curvature of the geostrophic streamfunction. Natural gradient winds are computed using the curvature of the streamlines of the actual wind or an observed parcel trajectory. If the geostrophic streamfunction or streamlines are not changing in time, the corresponding type of gradient wind is steady; otherwise, the gradient wind type is nonsteady. The kinds of gradient wind are summarized in Table 3, the contents of which reflect the findings discussed in the previous sections of this article. In the last column of the first two rows of Table 3, the disadvantage related to justification of the anomalous solution for the contour gradient wind assumes that no actual wind is available.

The gradient wind concept is generally accepted as an important teaching tool. To get some idea as to the prevalence of the utility of the gradient wind beyond the classroom, the author conducted a search for the phrase "gradient wind" in the abstracts of articles published in all American Meteorological Society (AMS) journals after 2006. The articles found were examined to determine the type of gradient wind used. If the determination of type was possible and decisive, the article is included in Table 4. Two noticeable aspects of Table 4 are the predominance of the steady contour gradient wind and its frequent application to tropical cyclones.
The prevalence of the steady contour gradient wind concept in tropical cyclone research has a long history. The application of gradient wind balance appears in early work on the topic of tropical cyclones and is justified with reference to scale analysis (e.g., Charney and Eliassen 1964). From early composite data studies (e.g., Gray and Shea 1973) and modeling studies (e.g., Emanuel 1986) to the contemporary work on tropical cyclones, the steady contour gradient wind continues to play an important role. A relatively recent article by Schwendike and Kepert (2008) gives an excellent justification for and description of applying the steady contour gradient wind to tropical cyclones. Given the preponderance of the steady contour gradient wind in the tropical cyclone arena, findings of supergradient winds are ubiquitous (e.g., Gray and Shea 1973; Bryan and Rotunno 2009; Frisius et al. 2013) as would be expected from the analysis and results presented in the previous sections of this article.

The geostrophic wind results from applying scale analysis to large-scale midtropospheric flow (Dutton 1986). The gradient wind, as a refinement of the geostrophic wind, is similarly applicable to large-scale flow. Yet, in many cases the gradient wind is applied on much smaller scales. For example, fine temporal and spatial resolution of Lagrangian trajectory data results in more accurate and applicable gradient wind estimates for parcels. Tropical cyclones are not large-scale phenomena from 
TABLE 4. Recent AMS articles mentioning "gradient wind" in the abstract and for which the type of gradient wind could be determined decisively. The entries are organized chronologically from earliest at top to most recent at bottom.

\begin{tabular}{lcc}
\hline \multicolumn{1}{c}{ Reference } & Type of gradient wind & Application \\
\hline Hodyss and Nolan (2007) & Steady contour & Warm or cold core vortices \\
Afanasyev et al. (2008) & Steady natural & Rotating tank simulations \\
Marks et al. (2008) & Steady contour & Tropical cyclones \\
Schwendike and Kepert (2008) & Steady contour & Tropical cyclones \\
Bryan and Rotunno (2009) & Steady contour & Tropical cyclones \\
Vickery et al. (2009) & Steady contour & Tropical cyclones \\
Forbes et al. (2010) & Steady contour & Tropical cyclones \\
Houze (2010) & Steady contour & Tropical cyclones \\
Kwon and Cheong (2010) & Steady contour & Tropical cyclones \\
Reed and Jablonowski (2011) & Steady contour & Tropical cyclones \\
Willoughby (2011) & Steady contour & General \\
Nakanishi and Niino (2012) & Steady contour & Tropical cyclones \\
Rotunno and Bryan (2012) & Steady contour & Tropical cyclones \\
Frisius et al. (2013) & Steady contour & Tropical cyclones \\
Wood et al. (2013) & Steady contour & Tropical cyclones \\
\hline
\end{tabular}

the midlatitude perspective; yet, the gradient wind is routinely applied to tropical cyclones (Table 4). In the opinion of the author, the large-scale context for the gradient wind is not necessary so long as the application of the gradient balance is justified in general by scale analysis or demonstrated to be valid for specific cases using real data.

In conclusion, the gradient wind remains an important concept of utility in teaching, as well as in research and practical applications (Table 4). If four people are given the same dataset with unqualified instructions to compute the gradient wind, they could return with four different values, all valid from the standpoint of the definition of the gradient wind. The primary goal of this article is to propose simple terminology that clearly distinguishes among the possible magnitudes of the gradient wind based on the method of computing the trajectory curvature for the gradient wind calculation. Contour gradient wind parcels follow trajectories based on the geostrophic streamfunction. Natural gradient wind parcels follow trajectories based on the actual wind field. In the case of steady flow of either type, the trajectories are streamlines; otherwise, Blaton's Eq. (1) must be included as in (4) to account for the local time tendency of the respective wind direction. The nonsteady natural gradient wind may be computed using the curvature obtained from successive parcel positions in the case of Lagrangian data or by computing the curvature directly applying (8) as described in section 3 . The nonsteady natural gradient wind magnitude provides an upper bound for the actual wind speed.

Acknowledgments. The insights of Dr. Daniel Keyser in an informal review of the draft manuscript were very valuable and much appreciated. The author is grateful to
Dr. Richard Rotunno, whose perusal of preliminary notes and informal review of a draft version resulted in many helpful suggestions. Reviews by Drs. David Novak and Wallace Hogsett yielded helpful suggestions for clarifying the presentation. Formal reviews by five anonymous reviewers and Dr. David Schultz were very helpful, resulting in improved and expanded content visà-vis the original manuscript. The encouragement and advice of Dr. Louis Uccellini are much appreciated. The National Centers for Environmental Prediction provided funding for this publication. Any opinions expressed in this article are solely those of the author and do not necessarily represent the position of the funding entity.

\section{APPENDIX}

\section{Demonstration of an Anticyclonic Curvature Limitation}

This demonstration shows that a supergradient wind speed is not possible if the gradient wind magnitude is that for the nonsteady natural case. Referring to (6) in the main text, which applies only to the nonsteady natural gradient wind, if $\left(V-V_{\mathrm{gr}}\right)=\delta>0$ and the parcel direction of motion is nearly aligned with the geostrophic wind, then (6) may be written as $\delta \approx-\delta\left(V_{\mathrm{gr}}+V\right) K^{\prime}$. This implies that $K^{\prime} \approx-1 /\left(V_{\mathrm{gr}}+V\right)$. However, a nonimaginary square root for the gradient wind equation requires $K^{\prime} \geq-1 / 4 V_{g}$. Even though $V_{g}<V_{\mathrm{gr}}$ for anticyclonic flow, the magnitude of $K^{\prime}$ needed for supergradient anticyclonic flow is nearly twice as large as the limit required for a nonimaginary square root. Thus, the assumption of supergradient wind for the nonsteady natural case leads to an impossible result for anticyclonic curvature. 


\section{REFERENCES}

Afanasyev, Y. D., P. B. Rhines, and E. G. Lindahl, 2008: Emission of inertial waves by baroclinically unstable flows: Laboratory experiments with altimetric imaging velocimetry. J. Atmos. Sci., 65, 250-262, doi:10.1175/2007JAS2336.1.

Bryan, G. H., and R. Rotunno, 2009: Evaluation of an analytical model for the maximum intensity of tropical cyclones. J. Atmos. Sci., 66, 3042-3060, doi:10.1175/2009JAS3038.1.

Carlson, T. N., 1980: Airflow through midlatitude cyclones. Mon. Wea. Rev., 108, 1498-1509, doi:10.1175/1520-0493(1980)108<1498: ATMCAT $>2.0 . \mathrm{CO} ; 2$.

Charney, J. G., and A. Eliassen, 1964: On the growth of the hurricane depression. J. Atmos. Sci., 21, 68-75, doi:10.1175/ 1520-0469(1964)021<0068:OTGOTH $>2.0 . \mathrm{CO} ; 2$

desJardins, M. L., K. F. Brill, and S. S. Schotz, 1991: Use of GEMPAK on UNIX workstations. Preprints, Seventh Int. Conf. on Interactive, Information and Processing Systems for Meteorology, Oceanography, and Hydrology, New Orleans, LA, Amer. Meteor. Soc., 449-453.

Dutton, J. A., 1986: The Ceaseless Wind. Dover Publications, Inc., $617 \mathrm{pp}$.

Emanuel, K. A., 1986: An air-sea interaction theory for tropical cyclones. Part I: Steady-state maintenance. J. Atmos. Sci., 43, 585-605, doi:10.1175/1520-0469(1986)043<0585 AASITF $>2.0 . \mathrm{CO} ; 2$.

Forbes, C., R. A. Luettich, C. A. Mattocks, and J. J. Westerink, 2010: A retrospective evaluation of the storm surge produced by Hurricane Gustav (2008): Forecast and hindcast results. Wea. Forecasting, 25, 1577-1602, doi:10.1175/2010WAF2222416.1.

Frisius, T., D. Schönemann, and J. Vigh, 2013: The impact of gradient wind imbalance on potential intensity of tropical cyclones in an unbalanced slab boundary layer model. J. Atmos. Sci., 70, 1874-1890, doi:10.1175/JAS-D-12-0160.1.

Fultz, D., 1991: Quantitative nondimensional properties of the gradient wind. J. Atmos. Sci., 48, 869-875, doi:10.1175/ 1520-0469(1991)048<0869:QNPOTG >2.0.CO;2.

Gray, W. M., and D. J. Shea, 1973: The hurricane's inner core region. II. Thermal stability and dynamic characteristics. J. Atmos. Sci., 30, 1565-1576, doi:10.1175/1520-0469(1973)030<1565: THICRI $>2.0 . \mathrm{CO} ; 2$.

Haltiner, G. J., and F. L. Martin, 1957: Dynamical and Physical Meteorology. McGraw-Hill Book Co., Inc., 470 pp.

Hodyss, D., and D. S. Nolan, 2007: Linear anelastic equations for atmospheric vortices. J. Atmos. Sci., 64, 2947-2959, doi:10.1175/ JAS3991.1.

Holton, J. R., 2004: An Introduction to Dynamic Meteorology. 4th ed. Elsevier Academic Press, 535 pp.
Houze, R. A., 2010: Clouds in tropical cyclones. Mon. Wea. Rev., 138, 293-344, doi:10.1175/2009MWR2989.1.

Kwon, I.-H., and H.-B. Cheong, 2010: Tropical cyclone initialization with a spherical high-order filter and idealized threedimensional bogus vortex. Mon. Wea. Rev., 138, 1344-1367, doi:10.1175/2009MWR2943.1.

Marks, F. D., P. G. Black, M. T. Montgomery, and R. W. Burpee, 2008: Structure of the eye and eyewall of Hurricane Hugo (1989). Mon. Wea. Rev., 136, 1237-1259, doi:10.1175/ 2007MWR2073.1.

Mogil, H. M., and R. L. Holle, 1972: Anomalous gradient winds: Existence and implications. Mon. Wea. Rev., 100, 709-716, doi:10.1175/1520-0493(1972)100<0709:AGWEAI>2.3.CO;2.

Nakanishi, M., and H. Niino, 2012: Large-eddy simulation of roll vortices in a hurricane boundary layer. J. Atmos. Sci., 69, 35583575, doi:10.1175/JAS-D-11-0237.1.

Reed, K. A., and C. Jablonowski, 2011: An analytic vortex initialization technique for idealized tropical cyclone studies in AGCMs. Mon. Wea. Rev., 139, 689-710, doi:10.1175/ 2010MWR3488.1.

Rotunno, R., and G. H. Bryan, 2012: Effects of parameterized diffusion on simulated hurricanes. J. Atmos. Sci., 69, 2284 2299, doi:10.1175/JAS-D-11-0204.1.

Schwendike, J., and J. D. Kepert, 2008: The boundary layer winds in Hurricanes Danielle (1998) and Isabel (2003). Mon. Wea. Rev., 136, 3168-3192, doi:10.1175/2007MWR2296.1.

Uccellini, L. W., R. A. Petersen, K. F. Brill, P. J. Kocin, and J. J. Tuccillo, 1987: Synergistic interactions between and upper-level jet streak and diabatic processes that influence the development of a low-level jet and a secondary coastal cyclone. Mon. Wea. Rev., 115, 2227-2261, doi:10.1175/ 1520-0493(1987)115<2227:SIBAUL >2.0.CO;2.

Vickery, P. J., D. Wadhera, M. D. Powell, and Y. Chen, 2009: A hurricane boundary layer and wind field model for use in engineering applications. J. Appl. Meteor. Climatol., 48, 381-405, doi:10.1175/2008JAMC1841.1.

Whitaker, J. S., L. W. Uccellini, and K. F. Brill, 1988: A modelbased diagnostic study of the rapid development phase of the Presidents' Day cyclone. Mon. Wea. Rev., 116, 2337-2365, doi:10.1175/1520-0493(1988)116<2337:AMBDSO > 2.0.CO;2.

Willoughby, H. E., 2011: The golden radius in balanced atmospheric flows. Mon. Wea. Rev., 139, 1164-1168, doi:10.1175/ 2010MWR3579.1.

Wood, V. T., L. W. White, H. E. Willoughby, and D. P. Jorgensen, 2013: A new parametric tropical cyclone tangential wind profile model. Mon. Wea. Rev., 141, 1884-1909, doi:10.1175/ MWR-D-12-00115.1. 